\title{
TESES E DISSERTACOOES SOBRE ENVELHECIMENTO PRODUZIDAS NA UNIVERSIDADE FEDERAL DO RIO GRANDE DO SUL E INCLUIDAS NO SISTEMA DE BIBLIOTECAS NO ANO DE 2012
}

\section{Sergio Antonio Carlos ${ }^{1}$}

O presente levantamento de teses e dissertações sobre envelhecimento produzidas na UFRGS e incluídas no sistema de Bibliotecas no ano de 2012, como os anteriores, foi realizado a partir do Catálogo Online (Sabi) e do Lume (repositório digital). Foram utilizados os seguintes descritores: Antienvelhecimento, Educação: pessoa idosa, Envelhecimento, Geriatria, Gerontologia, Idoso, Memória (quando relacionada com o envelhecimento ou com pessoa idosa), Odontologia Geriátrica, Pessoa Idosa, Qualidade de vida: idoso, Terceira Idade, Velhice.

Foram localizadas 20 (vinte) teses e dissertações defendidas nos PPGs de Administração, Ciências Biológicas: Bioquímica, Ciência do Movimento Humano, Ciências Médicas, Design, Direito, Economia, Educação, Enfermagem, Epidemiologia, Informática da Educação, Odontologia e Psicologia. A seguir apresentamos as referências - em ordem alfabética do sobrenome do autor - com a indicação para a localização do texto completo no Lume (Repositório Digital da UFRGS) - e respectivo resumo.

1 Editor da revista Estudos Interdisciplinares sobre o Envelhecimento, professor Associado do Instituto de Psicologia e coordenador do curso de Serviço Social. 
Exceção a uma dissertação do PPG de Psicologia que disponibilizou o resumo, mas não o texto; a uma tese e duas dissertações do PPG em Epidemiologia; uma dissetação do PPG Medicina: Ciências Médicas e uma tese (PPG Direito) que estão sem o resumo e a indicação para acesso ao texto porque estas informações não estão disponibilizadas no Sistema de Bibliotecas. As mesmas podem ser consultadas nas bibliotecas depositárias.

ALVARENGA, Luiz Fernando Calage. A arte de envelhecer ativamente: articulações entre corpo, gênero e sexualidade. 2012. 163 fl. Tese (doutorado) - Universidade Federal do Rio Grande do Sul. Faculdade de Educação. Programa de Pós-Graduação em Educação, Porto Alegre, BR-RS, 2012. Ori.: MEYER, Dagmar Elisabeth Estermann. Disponível em: http://hdl.handle. net $/ 10183 / 60400$

\section{resumo}

Esta tese tematiza relações que se estabelecem entre corpo, gênero, sexualidade e envelhecimento no contexto do que se define como envelhecimento saudável, na contemporaneidade. A pesquisa fundamentou-se nos estudos culturais e de gênero aproximados da perspectiva de Michel Foucault, em articulação com alguns estudos do envelhecimento. $O$ processo investigativo foi guiado pelas seguintes questões: como sujeitos ditos idosos/as estão (re) aprendendo a viver suas relações afetivas e sexuais na contemporaneidade e como corpo, gênero e sexualidade estão implicados nesses processos e com que efeitos? Para dar conta de tais questões foram realizados grupos focais e entrevistas semiestruturadas, dos quais participaram mulheres com idades entre 49 e 80 anos, integrantes de um programa voltado para a preparação para a aposentadoria na cidade de Caxias do Sul/RS. O material empírico produzido foi analisado na perspectiva da análise cultural. Utilizaram-se elementos da teorização foucaultiana sobre o cuidado de si, articulados com pressupostos de corpo, gênero e sexualidade como ferramentas analíticas; da análise realizada resultaram três unidades temáticas. A primeira toma como foco a articulação entre os processos de objetivação/ subjetivação operados na direção de reforçar a juventude como ideal a ser perseguido/aprendido continuamente. Na segunda discutem-se as (re)aprendizagens afetivas e sexuais nas quais os sujeitos estão envolvidos, dentro de determinados limites e possibilidades. Na terceira abordam-se os modos de pensar destes sujeitos com relação ao HIV/aids e as implicações disso em suas 
vidas e na vida daqueles com quem eles se relacionam. A análise realizada permite defender o argumento de que a sexualidade ativamente vivida é um dos imperativos associados ao envelhecimento esperado e indicado como saudável, na contemporaneidade. Para dar conta disso, sujeitos ditos idosos/ as precisam (re)aprender a viver suas relações afetivas e sexuais, sendo que esses processos são atravessados e dimensionados pelo gênero e têm o corpo como lócus central de investimentos.

palavras - chave

AIDS. Corpo. Envelhecimento. Gênero. Masculinidade. Sexualidade.

ARGENTA, Carla. Fatores de risco para a síndrome da fragilidade no idoso: contribuições para a elaboração de diagnósticos de enfermagem. 2012. 91 f.l: il. Dissertação (mestrado) - Universidade Federal do Rio Grande do Sul. Escola de Enfermagem. Programa de Pós-Graduação em Enfermagem, Porto Alegre, BR-RS, 2012. Ori.: CROSSETTI, Maria da Graça Oliveira. Disponível em: http://hdl.handle.net/10183/55412

\section{resumo}

A Síndrome da Fragilidade no Idoso (SFI) é entendida como uma síndrome clínica caracterizada pelo declínio funcional dos sistemas fisiológicos resultando na diminuição de energia e resistência do organismo. É determinada por fatores de risco sociais, biológicos, ambientais e psicológicos. O objetivo geral desta investigação foi: analisar os fatores de risco para a SFI visando contribuir para a elaboração de Diagnósticos de Enfermagem (DE) relacionados a esta condição clínica. Já os objetivos específicos foram caracterizar o perfil socioeconômico e demográfico, de morbidades crônicas e de condições de saúde dos idosos; identificar os fatores associados à SFI a partir do perfil socioeconômico e demográfico de morbidades crônicas e de condições de saúde dos idosos; verificar a prevalência da SFI a partir da aplicação da Escala de Fragilidade de Edmonton (EFS), verificar a associação entre os fatores de risco para SFI com os níveis da EFS e relacionar as características definidoras dos DE em idosos na comunidade e hospitalizados com as características dos fatores de risco para a SFI. Caracteriza-se por um estudo transversal com abordagem quantitativa, realizado com 306 idosos que frequentam 11 grupos de convivência do município de Frederico Westphalen/RS. Os dados foram coletados por meio de um instrumento contendo questões socioeconômicas 
e demográficas, morbidades crônicas e de condições de saúde com posterior aplicação da EFS. Para a análise dos dados, foi utilizado o programa estatístico SPSS versão 18.0. O projeto de pesquisa foi submetido ao Comitê de Ética da URI - campus de Frederico Westphalen, o qual foi aprovado e registrado sob o número CAEE 0046.0.284.000-11. Como resultados do estudo, constatouse, o predomínio do sexo feminino $196(64,1 \%)$ e da cor branca 249 (81,4\%), a maioria que vive com o cônjuge ou companheiro (a) 216 (70,8\%), e mora em casa própria 291 (95,1\%), reside ou residiu a maior parte de sua vida na área rural. Observa-se ainda que $272(89,2 \%)$ idosos sabem ler e estudaram de 1 a 4 anos $138(63,4 \%), 267(87,5 \%)$ idosos são aposentados. Constatou-se que 182 (59,5\%) idosos apresentaram HAS, 163 (54,5\%) Depressão, 100 (32,7\%) Doença do coração, 14 (4,6\%) Derrame cerebral, 40 (13,1\%) Problemas renais e 39 (12,8\%) Câncer e Diabetes Mellitus. Quanto às variáveis de condições de saúde, a presença de fraqueza no corpo foi referida por $141(46,2 \%)$ idosos e a queda no último ano ocorreu em $121(39,7 \%)$ idosos. Sendo assim, obtevese uma prevalência de SFI de 39,2\% (n=62), sendo que, dentre os sujeitos do estudo, 17,3\% (19) são do sexo masculino e 21,9\% (43) do sexo feminino. Os fatores de risco que permaneceram associados à SFI, após o ajuste pela análise multivariada, foram: idade $(p=0,002)$, fraqueza no corpo $(p=0,001)$, presença de morbidades crônicas $(p=0,042)$, convívio social $(p=0,046)$ e quedas $(p=0,011)$. De posse dos fatores de risco para a SFI, realizou-se uma relação entre as características definidoras de Diagnósticos de Enfermagem (DE) presentes em idosos na comunidade e hospitalizados presentes na literatura com as características dos fatores de risco para a SFI.

palavras - chave

Diagnóstico de enfermagem. Enfermagem geriátrica. Envelhecimento. Idoso fragilizado. Saúde do idoso.

BORGES, Roberto Cabral de Mello. Uso de tecnologias de informação e comunicação por adultos de idade avançada: considerações na área educacional. 2012. 202 fl. Tese (doutorado) - Universidade Federal do Rio Grande do Sul. Centro de Estudos Interdisciplinares em Novas Tecnologias da Educação. Programa de Pós-Graduação em Informática na Educação, Porto Alegre, BR-RS, 2012. Ori.: LIMA, Jose Valdeni de. Co-Ori.: REATEGUI, Eliseo Berni. Disponível em: http://hdl.handle.net/10183/53149 
resumo

Este trabalho pesquisa o uso das tecnologias de informação e comunicação que estão sendo usadas pelos adultos de idade avançada no Brasil. O objetivo principal é estudar o uso destas tecnologias para fins educacionais. Adultos idosos têm algumas limitações, têm dificuldades para operar os equipamentos e têm um comportamento particular a respeito do uso de algumas Tecnologias de Informação e Comunicação. Este estudo mostra como os adultos de idade mais avançada estão usando essas Tecnologias de Informação e Comunicação, suas dificuldades, seus sentimentos a respeito, suas expectativas e como eles estão obtendo algum aprendizado auxiliado por estas tecnologias e seus benefícios para sua qualidade de vida. Para designar este grupo de adultos, que abrange a $3^{\text {a }}$ idade e mais um segmento de idade imediatamente abaixo, foi criada a expressão "Adulto de Idade Avançada" (55 a 90 anos). Para que se possam quantificar as formas, intensidade de uso e dificuldades no uso foi criado um índice que mede o quanto e como estes adultos estão usando de cada tecnologia, permitindo assim uma comparação e classificação. Através desse índice podem-se determinar estatisticamente quais variáveis influenciam ou não o uso das tecnologias e inferir perfil e comportamento da população estudada. Conhecido este perfil pode-se fazer recomendações e associações de tecnologias com tipos de atividades que possam promover algum tipo de aprendizado para estes usuários e com isto lhes adicionar um componente a mais de qualidade de vida.

\section{palavras-chave}

Tecnologias de informação e comunicação (TICs). Terceira idade.

CARVALHO, Vivian Carla Honorato dos Santos de. Avaliação do Serviço de Atenção Básica à Saúde utilizado pela população idosa do município de Ilhéus/Bahia. 2012. 103 fl. Dissertação (mestrado profissional) - Universidade Federal do Rio Grande do Sul. Faculdade de Medicina. Programa de Pós-Graduação em Epidemiologia, Porto Alegre, BR-RS, 2012 Ori.: FUCHS, Sandra Cristina Pereira Costa

(Resumo e texto na íntegra não disponíveis on line)

palavras - chave

Doença. Idoso. Atenção primária à saúde. Saúde da família. 
COSTA, Marcelo Silveira da. O impacto da frequência do exercício físico sobre proteínas sinápticas e o comportamento de ratos durante a fase adulta e no envelhecimento. 2012. [125] fl.: il. Tese (doutorado) - Universidade Federal do Rio Grande do Sul. Instituto de Ciências Básicas da Saúde. Programa de PósGraduação em Ciências Biológicas: Bioquímica, Porto Alegre, BR-RS, 2012. Ori.: PORCIUNCULA, Lisiane de Oliveira. Disponível em: http://hdl.handle. net/10183/60988

\section{resumo}

Oexercício físico regulare/ou maiores níveis de atividade física tem contribuído para a redução do risco de doenças degenerativas e para melhorar a qualidade de vida. Estes benefícios são mais evidentes quando o corpo humano vai se tornando mais vulnerável, seja pelo processo natural de envelhecimento ou pelo acometimento de doenças. Entretanto, a quantidade adequada de exercícios físicos para a saúde e qualidade de vida ainda é muito discutida. Desta forma, o objetivo desta tese foi verificar o efeito da frequência semanal (1, 3 e 7 dias por semana) da corrida em esteira (20 minutos por sessão, intensidade moderada) em ratos durante a fase adulta e no envelhecimento. No primeiro trabalho foi observado um comportamento ansiogênico associado ao envelhecimento. Entretanto, a frequência de 1 dia por semana foi capaz de reverter esse efeito da idade. Todas as frequências de corrida em esteira causaram um aumento na ansiedade nos ratos adultos. Não encontramos nenhuma relação da ansiedade com o imunoconteúdo hipocampal dos receptores de adenosina A1 e A2A. Entretanto, o treinamento em esteira foi capaz de reverter o aumento no imunoconteúdo do A2A mediada pelo envelhecimento, sendo o efeito da corrida realizada 7 dias/semana mais pronunciado. Este efeito sobre o receptor A2A pode estar associado aos aspectos neuroprotetores do exercício físico. No segundo trabalho, observamos que o exercício físico não afetou a memória de curta e longa duração nas tarefas de reconhecimento de objetos e esquiva inibitória nos ratos adultos. Porém, as memórias de curta e longa duração foram prejudicadas pela idade na tarefa de reconhecimento de objetos, mas a corrida em esteira realizada 1 dia/semana reverteu esse efeito. A idade causou uma redução no desempenho da memória de curta duração na tarefa de esquiva inibitória. A corrida realizada 1 dia/semana prejudicou as memórias de curta e longa duração nesta tarefa nos ratos de meia idade. A idade causou um aumento no imunoconteúdo hipocampal de BDNF com uma concomitante redução de seu receptor, TrkB, o que está relacionado com o prejuízo cognitivo associado à idade. Foi encontrado um aumento 
no precursor do BDNF, pró-BDNF, com o treinamento realizado 7 dias por semana nos animais de meia idade, mas um ligeiro aumento no TrkB no grupo treinado 1 dia/semana poderia explicar a reversão do prejuízo da memória de reconhecimento associada à idade. Nos ratos adultos foi encontrado um aumento do pró-BDNF e do BDNF no grupo treinado 1 dia/semana e uma redução do TrkB em todos os grupos submetidos à corrida em esteira. Apesar destas alterações decorrentes do exercício físico nos ratos adultos não influenciarem no desempenho das tarefas de aprendizagem e memória, a redução do TrkB poderia ter contribuído para que os animais submetidos ao treinamento físico não apresentassem desempenhos cognitivos superiores ao grupo sedentário. Assim sendo, a frequência da corrida em esteira e a idade afetaram as respostas nas tarefas de memória e na adaptação neurotrófica no hipocampo. O trabalho 3 verificou o efeito de praticar exercício físico apenas um dia/ semana até à exaustão versus 3 dias/semana em intensidade moderada em ratos adultos. A corrida em esteira causou um comportamento ansiogênico, independente do protocolo de exercício. A corrida realizada 1 dia/semana até a exaustão causou uma redução no comportamento exploratório e locomotor e um prejuízo na memória de reconhecimento. Desta forma, a prática de exercícios físicos apenas um dia/semana até à exaustão altera o comportamento e prejudica a memória. Em conclusão, esta tese mostra a importância do controle da frequência semanal do exercício físico para um melhor entendimento das respostas comportamentais e neuroquímicas mediadas pelo exercício físico crônico em diferentes idades.

palavras - chave

Ansiedade. Comportamento animal. Exercício Físico. Memória. Neuroquímica. Ratos.

GAMARRA, Ariane Haydée Estrada. Análise neurofisiológica do equilíbrio através da eletromiografia durante a realização do pull test em indivíduos normais. 2012. 87fl. Dissertação (mestrado) - Universidade Federal do Rio Grande do Sul. Faculdade de Medicina. Programa de Pós-Graduação em Medicina: Ciências Médicas, Porto Alegre, BR-RS Ori.: Rieder, Carlos Roberto de Mello. Co-Ori.: SCHESTATSKY, Pedro.

(Resumo e texto na íntegra não disponíveis on line) 
Doença. Eletromiografia. Envelhecimento. Equilibrio Postural. Cognição.

LINN, Luiziane Brusa da Costa. "Essas pessoas que envelhecem...": saberes de adultos com deficiencia intelectual. 2012. 121 fl. Dissertação (mestrado) - Universidade Federal do Rio Grande do Sul. Faculdade de Educação. Programa de Pós-Graduação em Educação, Porto Alegre, BR-RS, 2012. Ori.: DOLL, Johannes. Disponível em: http://hdl.handle.net/10183/61972

\section{resumo}

Compreendendo a velhice como categoria socialmente construída através dos processos de socialização estabelecidos pelo indivíduo ao longo do curso de sua vida e que vão lhe permitir construir sua identidade, considera-se o envelhecimento como um processo individual, entendido e vivenciado por cada um desde o lugar e o papel que a sociedade lhe destina. Partindo dessas concepções, pensar num grupo que tem sua realidade pautada pela exclusão social (do mundo das relações, da escola, do trabalho, etc) implica buscar conhecer como essas pessoas estão percebendo e vivenciando o processo de envelhecimento. Nesta pesquisa, um grupo de adultos com deficiência intelectual nos fala de suas experiências com o processo de envelhecimento e suas imagens da velhice. Por meio da Observação Participante e da técnica do Grupo Focal adaptada às particularidades do grupo, se buscou conhecer os seus saberes e representações da velhice e do processo de envelhecimento. Este estudo pôde se aproximar da construção do conhecimento desse grupo sobre a velhice e o processo de envelhecimento, passando pelas particularidades que marcam o curso de vida dos integrantes do grupo e as construções de si mesmos enquanto sujeitos sociais. Pois é a partir do lugar e papel atribuídos aos sujeitos com deficiência intelectual em nossa sociedade que eles constroem as suas experiências de envelhecimento e é a partir desse lugar que enxergam e nos falam sobre a velhice. É necessário salientar que embora se aborde uma discussão sobre o processo de envelhecimento no contexto da deficiência intelectual, a pesquisa trata de discutir essa questão em um grupo específico de indivíduos, não cabendo generalizações, visto que tanto as maneiras de envelhecer quanto as experiências com a deficiência intelectual são bastante distintas e diversas, e envolvem os mais diversos determinantes.

palavras - chave

Deficiência intelectual. Grupo focal. Velhice. 
LOCATELLI, Patrícia Augusta Pospichil Chaves. As representações sociais sobre a velhice e os reflexos nos processos de gestão de pessoas de uma instituição de longa permanência de Porto Alegre. 2012. 230 fl.: il. Dissertação (mestrado) - Universidade Federal do Rio Grande do Sul. Escola de Administração. Programa de Pós-Graduação em Administração, Porto Alegre, BR-RS, 2012. Ori.: CAVEDON, Neusa Rolita. Disponível em: http://hdl.handle. net $/ 10183 / 49412$

resumo

Nas últimas décadas, o envelhecimento da população brasileira tem demandado atenção, principalmente, no que tange ao cuidado para com o público idoso. Apesar de a legislação brasileira estabelecer que o principal responsável pelo cuidado ao idoso é a família, a dinamicidade do contexto social e a atual fluidez dos relacionamentos familiares tem reconfigurado essa prerrogativa. Neste contexto, com a finalidade de suprir essa demanda oferecendo ao público longevo serviços de assistência social e à saúde, principalmente, em casos em que o idoso não possui família ou esta não apresenta condições para assumir a responsabilidade pelo seu cuidado, surgem as denominadas Instituições de Longa Permanência para Idosos - ILPIs, objeto deste estudo. Partindo-se do entendimento de que a forma como os funcionários de uma ILPI são geridos e a prestação de serviços conduzida está diretamente relacionada às representações sociais de gestores, funcionários e dos próprios idosos sobre a velhice, este estudo objetivou identificar e analisar como as representações sociais sobre a velhice na ótica dos usuários e daqueles que trabalham em uma instituição de longa permanência para idosos se refletem nos processos de movimentação e desenvolvimento de pessoas dessa instituição. Para isso, esta pesquisa, de abordagem qualitativa e natureza exploratório-descritiva, adotou como estratégia o estudo de caso e como técnicas de coleta de dados: observação simples e participante, realizadas de setembro de 2011 a fevereiro de 2012; entrevistas semiestruturadas com 18 indivíduos, sendo quatro gerentes, nove funcionários e cinco idosos; classificação de fotos; pesquisa documental. Os resultados deste estudo apontaram para a heterogeneidade das representações sociais de gestores, funcionários e usuários da instituição pesquisada a respeito da velhice, que perpassaram não apenas o contexto de institucionalização, mas também os dois modelos predominantes de velhice, um com foco nas perdas e outro com foco nos ganhos. A análise dessas representações sociais permitiu ainda o desvelamento de questões pertinentes à dinâmica organizacional como as relacio- 
nadas a gênero, relações de poder, mecanismos de controle da velhice e mecanismos de resistência. Quanto aos processos de movimentação e desenvolvimento de pessoas, identificouse que as representações sociais a respeito da velhice influenciam na condução das ações relacionadas aos processos de captação, internalização e desenvolvimento de pessoas, se desdobrando na filosofia de atendimento da ILPI pesquisada e no comportamento de seus gestores e funcionários.

palavras-chave

Gestão de pessoas. Representações sociais. Velhice.

MESQUITA, Riovaldo Alves de. Ensaios sobre seguridade social no Brasil. 2012. 235 fl.: il. Tese (doutorado) - Universidade Federal do Rio Grande do Sul. Faculdade de Ciências Econômicas. Programa de Pós-Graduação em Economia, Porto Alegre, BR-RS, 2012. Ori.: BALBINOTTO NETO, Giacomo. Disponível em: http://hdl.handle.net/10183/70013

\section{resumo}

O objetivo geral é analisar os efeitos da dinâmica demográfica e do crescimento econômico sobre a sustentabilidade da Seguridade Social no Brasil. No primeiro dos três ensaios, é analisado o impacto do risco de longevidade na previdência complementar fechada, bem como as possíveis consequências sociais e econômicas do envelhecimento populacional. O risco de longevidade pode ser absorvido pela Patrocinadora, transferido para o participante ou transferido para o mercado, e as vantagens e desvantagens de cada uma dessas estratégias são discutidas. No segundo, ensaio a sustentabilidade da Previdência Social no século XXI é analisada sob a ótica da dinâmica demográfica e do crescimento da produtividade. Argumenta-se que a sustentabilidade não depende de superávits contábeis, mas da manutenção da trajetória temporal do custo dentro da capacidade de financiamento do Governo. Por meio de um modelo de simulações, são criadas 440 trajetórias de custo para os benefícios de Aposentadoria por Tempo de Contribuição, Aposentadoria por Idade, Aposentadoria por Invalidez, Pensão por Morte e o benefício de prestação continuada de Assistência Social ao Idoso, entre 2012 e 2100. As simulações são analisadas quanto aos efeitos que as mudanças demográficas, de crescimento da produtividade, de condições de elegibilidade e das condições de reajustes dos benefícios têm sobre a trajetória de custo. $\mathrm{O}$ 
terceiro ensaio utiliza os mesmos cenários simulados, benefícios e horizonte temporal do segundo ensaio, mas seu foco de análise é a trajetória de contribuição das coortes de trabalhadores, expressa como percentual da renda per capita. São simuladas, ao todo, 1.800 trajetórias de contribuição. Conclui-se que, sob taxas de crescimento da produtividade próximas à média histórica do período 1900/2010 e com o envelhecimento populacional projetado para as próximas décadas, as condições atuais de elegibilidade e de reajuste dos benefícios são insustentáveis. Os resultados conjuntos dos três ensaios apontam para a inevitabilidade do envelhecimento populacional brasileiro e para a necessidade de que a Seguridade Social e se adapte a esse fenômeno.

\section{palavras - chave}

Brasil. Crescimento econômico. Demografia. Envelhecimento da população. Previdência complementar. Previdência social. Seguridade social.

NEVES, Matheus. Associação entre resiliência e satisfação com os serviços odontológicos acessados por idosos. 2011. 97 fl.: il. Dissertação (mestrado) - Universidade Federal do Rio Grande do Sul. Faculdade de Odontologia. Programa de Pós-Graduação em Odontologia, Porto Alegre, BR-RS, 2011. Ori.: HUGO, Fernando Neves. Disponível em: http://hdl.handle.net/10183/56467

\section{resumo}

O perfil epidemiológico da população idosa brasileira evidencia um quadro de extensas perdas dentárias e problemas de saúde bucal. A fim de reverter este quadro, observa-se o desenvolvimento de diversas políticas públicas de saúde cujo objetivo está em garantir atenção integral à saúde da população idosa, com ênfase no envelhecimento saudável e ativo. Porém, no que diz respeito aos Serviços de Saúde Bucal, poucos estudos têm sido desenvolvidos na ambição de conhecer a satisfação deste grupo etário com tais serviços. $\mathrm{O}$ objetivo deste estudo foi investigar a associação entre a resiliência e a satisfação com os Serviços Odontológicos, levando em consideração variáveis exógenas, determinantes primários, comportamentos e condições de saúde. Investigou-se a hipótese de que a resiliência está associada à satisfação com os Serviços Odontológicos acessados pelos idosos. O lócus da pesquisa foi a gerência distrital Lomba-Partenon, em Porto Alegre - RS, onde 771 idosos foram identificados em seus domicílios por meio de amostragem por conglomerado. Os indivíduos responderam a um questionário sócio-demográfico e 
de comportamentos em saúde, à Escala de Resiliência e a questões relativas ao Serviço Odontológico acessado e à satisfação com o mesmo; além disso, foi realizado um breve exame bucal para contagem do número de dentes e identificação do uso de prótese dentária. Baseado em uma abordagem hierárquica realizada através de Regressão Logística Multivariada, as odds ratios estimadas das variáveis que ficaram significativamente associadas com o desfecho em estudo, satisfação com o Serviço Odontológico, após a análise totalmente ajustada, foram: 1) obtenção de consulta odontológica classificada como regular: $\mathrm{OR}=1,85,95 \%$ IC $(1,10$ a 3,12); 2$)$ obtenção de consulta odontológica classificada como ruim: $\mathrm{OR}=2,17,95 \%$ IC $(1,05$ a 4,50$)$ e 3$)$ alto potencial de resiliência: $\mathrm{OR}=0,60,95 \%$ IC $(0,37$ a 0,97$)$. Portanto, os resultados confirmam a hipótese de associação entre elevado potencial de resiliência e satisfação com os Serviços Odontológicos acessados por idosos.

\section{palavras - chave}

Comportamento humano. Odontologia geriátrica. Qualidade de vida.

OLIVEIRA, Elise Botteselle de. Avaliação da qualidade do cuidado a idosos nos serviços da rede pública de atenção primária de Porto Alegre. 2012126 fl. Dissertação (mestrado profissional) - Universidade Federal do Rio Grande do Sul. Faculdade de Medicina. Programa de Pós-Graduação em Epidemiologia, Porto Alegre, BR-RS, 2012 Ori.: Bozzetti, Mary Clarisse. Co-Ori.: HARZHEIM, Erno.

(Resumo e texto na íntegra não disponíveis on line)

\section{palavras - chave}

Doença. Atenção primária à saúde. Envelhecimento. Avaliação de serviços de saúde.

PEREIRA, Gabriela Fonseca. O design com segurança e conforto no projeto de camas para a terceira idade. 012.140 fl.: il., fots. Dissertação (mestrado) - Universidade Federal do Rio Grande do Sul. Escola de Engenharia. Faculdade de Arquitetura. Programa de Pós-Graduação em Design, Porto Alegre, BR-RS, 2012. Ori.: BATISTA, Vilson Joao. Co-Ori.: AMARAL, Fernando Goncalves. Disponível em: http://hdl.handle.net/10183/60720 
A população idosa está em crescimento no Brasil segundo dados do último Censo Demográfico do Instituto Brasileiro de Geografia e Estatística - IBGE. Com esta perspectiva é importante atentar-se para o fato de que durante o processo de envelhecimento ocorrem diversas mudanças físicas e psíquicas que influenciam a pessoa no uso dos espaços e dos produtos. Dentre esses produtos, a cama é extremamente necessária durante toda a vida e a sua utilização faz parte da rotina diária, sendo alta a ocorrência de quedas de idosos no uso deste produto. Embora as lesões e as quedas na utilização das camas não estejam diretamente associadas à terceira idade, neste período da vida, elas podem representar um problema de saúde mais grave. A intenção desta dissertação é a proposição de diretrizes projetuais para que as camas sejam seguras e confortáveis para os idosos. Para alcançar este objetivo e compreender as necessidades dos idosos desenvolveramse duas etapas distintas: a fundamentação teórica e a pesquisa de campo. Na fundamentação teórica, foram levantados os assuntos relevantes a oito temas principais: idosos, mercado de camas e colchões, segurança, conforto, desenho universal, ergonomia, antropometria e usabilidade, com a finalidade de apontar direcionamentos de projeto indicados pelos autores. A pesquisa de campo, realizada em uma instituição de longa permanência e dezenove residências, totalizando cinquenta idosos, contou com o método de estudo de casos múltiplos através da aplicação de entrevistas semi-estruturadas entre idosos e profissionais. A aplicação deste método auxiliou a elaboração das diretrizes, pois, além de identificar as dificuldades encontradas pelos idosos na utilização da cama, permitiu a identificação dos elementos que são mais importantes e os que necessitam de melhoria na opinião dos usuários. As entrevistas com os profissionais que lidam com idosos diariamente também auxiliou neste processo. Assim, a partir da sistematização dos dados obtidos na fundamentação teórica e na pesquisa de campo, são apresentadas diretrizes projetuais que visam a segurança e ao conforto do idoso, que possui a sua autonomia preservada, na utilização da cama.

palavras - chave

Cama. Design. Design de produto. Idoso

PETRY, Diogo Miranda. Satisfação de vida, cognição e exercício físico em idosos praticantes de exercícios físicos. 2012. 73 fl. Dissertação (mestrado) - Universidade Federal do Rio Grande do Sul. Faculdade de Medicina. Programa de 
Pós-Graduação em Medicina: Ciências Médicas, Porto Alegre, BR-RS, 2012. Ori.: CHAVES, Marcia Lorena Fagundes. Disponível em: http://hdl.handle. net/10183/61260

\section{resumo:}

O objetivo do presente estudo foi determinar as características sóciodemográficas, cognitivas (memória, atenção, funções executivas, linguagem), satisfação de vida e perfil da atividade física (tipo e duração) de idosos praticantes de exercício. Além disso, o desempenho cognitivo e avaliação da satisfação de vida de indivíduos idosos fisicamente ativos foram comparados com os idosos não-ativos. Métodos: Uma avaliação observacional transversal foi realizado com idosos com idade acima de 60 anos, divididos em ativos fisicamente $(\mathrm{n}=$ 42) e fisicamente não-ativos ( $n=33)$, de acordo com a escala Centro Espacial Johnson. Dados demográficos, estado cognitivo (MMSE), sintomas depressivos (GDS-15), a satisfação de vida (SWLS), fluência verbal categórico (FAS e Animal), tarefa Dígitos (direto e inverso), habilidades linguísticas (Boston Naming Test), e memória episódica (memoria, lembrança e reconhecimento da Lista de palavras - CERAD) foram avaliados em ambos os grupos. Análise descritiva (média e desvio padrão) foi calculada para os dados sociodemográficos, MSSE, GDS-15, SWLS, e todos os testes cognitivos. ANOVA de uma via com correção de Bonferroni foi utilizado para as análises entre os grupos e para controlar as variáveis de confusão (escolaridade e idade). Uma análise do sub-conjunto foi realizado com grupos pareados pelo educação $(n=33$, cada), já que a educação foi significativamente diferente entre os grupos e afetando fortemente os resultados.) Resultados: o desempenho no teste de fluência verbal (FAS-F, p <0,005; FAS-S, p <0,005), teste de nomeação de Boston ( $\mathrm{p}$ $<0,005)$, e reconhecimento de lista de palavra do CERAD ( $<<0,001)$ do grupo fisicamente ativo foi maior do que o grupo nãoativo. Não foi observada diferença significativa entre os grupos em outras avaliações de funções cognitivas e de satisfação com a vida. Nas análises com a formação de grupos pareados, desempenho no teste de reconhecimento de lista de palavras do CERAD ( $p$ $<0,001)$ foi estatisticamente diferente entre os grupos. Aqueles que eram fisicamente ativos apresentaram escores mais elevados $(p<0,000)$. Conclusão: Os idosos que eram fisicamente mais ativos na vida mostraram desempenho melhor no reconhecimento de memória do que os não-ativos indivíduos. Não houve relação com a satisfação com a vida presente nessa amostra.

palavras - chave

Cognição. Envelhecimento. Exercício. Idoso. Qualidade de vida. 
SANT ANNA, Gabriela dos Santos. Efeito do extrato etanólico de curcuma longa sobre a atividade da enzima histona desacetilase no processo de envelhecimento cerebral. 2012. 63 fl. Dissertação (mestrado) - Universidade Federal do Rio Grande do Sul. Faculdade de Medicina. Programa de Pós-Graduação em Medicina: Ciências Médicas, Porto Alegre, BR-RS, 2012. Ori.: SIQUEIRA, Ionara Rodrigues. Disponível em: http://hdl.handle.net/10183/69642

\section{resumo}

Com o aumento da expectativa de vida, estima-se um aumento na incidência de doenças neurodegenerativas, como a Doença de Alzheimer (DA). Vários processos fisiopatológicos interligados estão envolvidos tanto no processo do envelhecimento, quanto na etiologia das desordens neurodegenerativas, como por exemplo, o estresse oxidativo e a neuroinflamação. Têm sido descrito que mecanismos epigenéticos podem estar relacionados com o processo de envelhecimento, ocasionando modificações na expressão gênica em diferentes regiões do cérebro, como por exemplo, a acetilação de histonas que é responsável em parte, pela transcrição gênica, sendo esse processo controlado por duas enzimas, a histona acetiltransferase (HAT) e a histona desacetilase (HDAC). A HAT é responsável por ocasionar um relaxamento da cromatina e com isso uma maior acessibilidade para o processo de transcrição gênica enquanto que a HDAC causa uma maior compactação da cromatina, resultando uma supressão na transcrição gênica. Considerando o papel da acetilação de histonas no processo de envelhecimento, em especial em doenças neurodegenerativas, tem sido sugerido o uso de estratégias que aumentem a acetilação com finalidade neuroprotetora como, por exemplo, o uso de inibidores de HDAC. Diversos estudos demonstram que os inibidores da HDAC são capazes de melhorar as funções cognitivas e a formação de memória. Nesse contexto, a curcumina, um composto polifenólico encontrado no açafrão (Curcuma longa) tem demonstrado ser capaz de atuar sobre os mecanismos epigenéticos, sendo sugerido como um potente inibidor da HDAC.Assim, o objetivo desse trabalho foi investigar o tratamento agudo com extrato etanólico de Curcuma longa sobre a atividade da enzima histona desacetilase em estruturas cerebrais, hipocampo e córtex frontal, de ratos Wistar. Ratos Wistar machos adultos (3 e 20 meses de idade) foram aleatoriamente distribuídos nos seguintes grupos: salina, veículo (DMSO), extrato etanólico de Curcuma longa $10 \mathrm{mg} / \mathrm{Kg}$ e $50 \mathrm{mg} / \mathrm{Kg}$. A atividade da histona desacetilase (HDAC) foi mensurada após 2 e 18 horas da administração, utilizando kit de ELISA específico. A ANOVA de três vias indicou um efeito 
do tempo $(\mathrm{F}(1,71)=4.238, \mathrm{p}=0.044)$ após a administração do extrato etanólico de Curcuma longa e um efeito da idade em hipocampos $(\mathrm{F}(1,71)=10.430$, $\mathrm{p}=0.002$ ), ou seja, animais com 20 meses apresentaram atividade maior da HDAC quando comparados com os animais de 3 meses de idade. Em córtex frontal, a ANOVA de três vias indicou um efeito da idade $(\mathrm{F}(1,75)=4.438, \mathrm{p}=0.039)$ e uma interação entre idade e tempo $(\mathrm{F}(1,75)=1.765, \mathrm{p}=0.001)$. Em ambas as estruturas analisadas, não houve efeito da administração com extrato de Curcuma longa. Nossos resultados demonstram que a atividade da histona desacetilase foi aumentada em ambas as estruturas testadas de ratos com 20 meses de idade, sugerindo que no processo de envelhecimento cerebral ocorra uma redução na acetilação de histonas, o que pode reduzir a transcrição gênica. Além disso, a atividade da HDAC parece ser influenciada pelo ritmo circadiano, uma vez que houve um efeito do tempo nos níveis da atividade da HDAC em ambas as estruturas analisadas. A atividade da HDAC foi maior no tempo de 18 horas (correspondendo ao período da manhã) quando comparado ao tempo de 2 horas, que corresponde ao turno da tarde, este dado pode estar relacionado à cronobiologia, onde a organização circadiana permite que o organismo mantenha a homeostase em resposta a variações diárias decorrentes do ambiente externo e do próprio organismo. Cabe descrever que os ratos Wistar são notívagos, onde o período da manhã é o início do período de sono; assim poderíamos sugerir uma redução da acetilação e consequentemente uma redução na expressão gênica nesse período.

\section{palavras - chave}

Cortex cerebral. Curcuma. Envelhecimento. Hipocampo. Histona. Ratos Wistar.

SCHMITT, Cristiano Heineck. A "hipervulnerabilidade" do idoso no âmbito do direito fundamental de proteção do consumidor. 2012. 306 fl. Tese (doutorado) - Universidade Federal do Rio Grande do Sul. Faculdade de Direito. Programa de PósGraduação em Direito, Porto Alegre, BR-RS, 2012. Ori.: OLIVEIRA JÚNIOR, José Alcebíades de.

(Resumo e texto na íntegra não disponíveis on line)

palavras - chave

Direito. Proteção ao consumidor. Direito do consumidor. Direito do idoso. 
SERBIM, Andreivna Kharenine. Redes e apoio social percebido por idosos usuários de um serviço de emergência. 2012. 122 fl. Dissertação (mestrado) - Universidade Federal do Rio Grande do Sul. Escola de Enfermagem. Programa de PósGraduação em Enfermagem, Porto Alegre, BR-RS, 2012. Ori.: PASKULIN, Lisiane Manganelli Girardi. Disponível em: http://hdl.handle.net/10183/49101

\section{resumo}

Os objetivos deste estudo longitudinal foram: analisar a rede de apoio formal e informal e o apoio social percebido por idosos usuários do serviço de emergência do HCPA; caracterizar a amostra segundo aspectos sociodemográficos, de saúde e de apoio; identificar as fontes de apoio e tipo de relacionamento do idoso com a fonte; verificar associações entre a média do apoio social percebido e as seguintes variáveis sociodemográficas: idade, sexo, escolaridade, número de filhos vivos, número de pessoas residentes no domicílio, estado conjugal e arranjo familiar; comparar a rede de apoio e o apoio social recebido e fornecido, citados pelos idosos que permaneceram hospitalizados, no momento do atendimento na emergência e duas semanas após a alta hospitalar. A amostra foi de 220 idosos, que utilizaram o serviço de emergência do HCPA. Para a coleta de dados, foram utilizados o Mini Exame do Estado Mental (MEEM), o Inventário da Rede de Suporte Social (IRSS) e um questionário estruturado que caracterizava os aspectos sociodemográficos, de saúde e apoio social. Entre os respondentes 51,8\% eram do sexo feminino, a média de idade foi de 71,6 anos ( $\mathrm{DP}=8,1), 57,3 \%$ eram procedentes do município de Porto Alegre, 42,7\% eram casados, 68,2\% eram aposentados, 40,9\% tinham até 4 anos de estudo e 64,6\% tinha renda familiar de até 2 salários-mínimos. Os idosos tinham 3,5 filhos e 2,9 pessoas na residência em média, 76,3\% vivia com a família. Os respondentes declararam ter 1,8 morbidades em média, $48,6 \%$ relataram ter a saúde ruim ou muito ruim e $43,6 \%$ citaram as doenças do aparelho circulatório como grupo de morbidades. Entre os idosos, 90,2\% relataram receber apoio da família, 81,5\% relataram fornecer apoio para a família e $72,3 \%$ recebiam apoio de uma instituição formal. O tamanho da rede de apoio foi de 4,8 em média e o escore de apoio social percebido foi de $4,2(\mathrm{DP}=0,4)$. A avaliação da rede de apoio permitiu conhecer os vínculos e as relações que constituem as redes de apoio dos usuários, em um momento de agravo nos quais mais necessitavam de apoio de uma rede formal e informal, propiciando aos profissionais de saúde explorar esses recursos. Estudos sobre redes de apoio podem auxiliar o planejamento em saúde no sentido de propi- 
ciar a reorganização dos sistemas, visualizando quais pessoas e instituições fazem parte dessa rede e como os usuários idosos percebem o apoio social.

palavras-chave

Apoio social. Emergências. Saúde do idoso. Serviço hospitalar de emergência.

TAVARES, Noemia Urruth Leão. Adesão ao tratamento em idosos e hipertensos. 2012136 fl. Tese (doutorado) - Universidade Federal do Rio Grande do Sul. Faculdade de Medicina. Programa de Pós-Graduação em Epidemiologia, Porto Alegre, BR-RS, 2012 Ori.: MENGUE, Sotero Serrate.

(Resumo e texto na íntegra não disponíveis on line)

\section{palavras - chave}

Doença. Adesão à medicação. Hipertensão. Idoso. Atenção primária à saúde. Estudos transversais.

VALER, Daiany Borghetti. Adaptação do instrumento Caregiver Burden Inventory para uso com cuidadores de pessoas idosas no Brasil. 2012. 107 fl. Dissertação (mestrado) - Universidade Federal do Rio Grande do Sul. Escola de Enfermagem. Programa de Pós-Graduação em Enfermagem, Porto Alegre, BR-RS, 2012. Ori.: PASKULIN, Lisiane Manganelli Girardi. Disponível em: http:// hdl.handle.net/10183/49105

\section{resumo}

Esta dissertação de mestrado versa sobre o processo de adaptação transcultural de um instrumento que avalia a sobrecarga de cuidadores de pessoas idosas. Está integrada a um projeto maior sobre responsabilidade filial no cuidado aos pais idosos. Este trabalho teve como objetivo adaptar o instrumento Caregiver Burden Inventory para uso com cuidadores familiares principais de pessoas idosas no Brasil. Foi realizado um estudo metodológico que compreendeu as etapas de tradução inicial, síntese das traduções, retro tradução, comitê de especialistas, pré-teste e submissão dos documentos aos autores. O comitê foi composto por cinco profissionais, médicos e enfermeiros, com experiência em atenção básica e saúde do idoso, sendo que três apresentavam experiência em adaptação de instrumentos e dois domínio da 
língua inglesa. O pré-teste foi realizado com oito cuidadores familiares principais de idosos com dependência para realizar uma ou mais Atividades da Vida Diária, vinculados ao Programa de Atendimento Domiciliar da Unidade Básica de Saúde Santa Cecília do Hospital de Clínicas de Porto Alegre. Estes eram na maioria mulheres $(\mathrm{n}=7)$, com uma média de 48 anos de idade e 11,5 anos de estudo. A análise dos dados se deu por meio da descrição do processo de adaptação do instrumento e da comparação com outros estudos. A primeira etapa do processo de adaptação resultou em duas traduções independentes do instrumento para a língua portuguesa falada no Brasil (T1 e T2). Na etapa seguinte, as discrepâncias existentes entre as traduções foram evidenciadas e discutidas pelas tradutoras e a pesquisadora, sendo elaborada consensualmente uma versão síntese das traduções (T3). Esta, por sua vez, foi submetida à retro tradução por dois profissionais, de forma cega em relação às traduções, resultando em duas versões retrovertidas para a língua inglesa (RT1 e RT2). O comitê de especialistas analisou as equivalências semântica, idiomática, experimental e conceitual entre as versões obtidas (T1, T2, T3, RT1 e RT2) e o instrumento original e, mediante consenso, compôs uma versão préfinal em português que foi submetida ao pré-teste. Foram verificadas dúvidas dos cuidadores em relação a três itens do instrumento, discutidos posteriormente com o comitê de especialistas que, mediante consenso, compôs uma versão final em português. A mesma foi retro traduzida para a língua inglesa e enviada ao autor da escala original, que aprovou a versão. Com a adaptação transcultural foi possível compor uma versão final do instrumento que preservou as equivalências quando comparado à versão original. Ainda que seja necessário testar as propriedades psicométricas, o processo mostrou que o instrumento possui itens e domínios relevantes para a análise da sobrecarga do cuidador no contexto local.

\section{palavras-chave}

Cuidadores idoso. Saúde do idoso.

ZABALETA, Ariane Silveira Dias. Velhice e lazer: um olhar etnográfico para um projeto de idosos. 2012. 103 fl.: il. Dissertação (mestrado) - Universidade Federal do Rio Grande do Sul. Escola de Educação Física. Programa de PósGraduação em Ciências do Movimento Humano, Porto Alegre, BR-RS, 2012. Ori.:STIGGER, Marco Paulo. Disponível em: http://hdl.handle.net/10183/63161 
Nesse trabalho me propus a compreender o que significa e como se vivencia a velhice dentro do Centro de Esporte, Lazer e Recreação do Idoso (CELARI), localizado na Escola de Educação Física da Universidade Federal do Rio Grande do Sul, em Porto Alegre (RS). A partir de uma incursão etnográfica (observação participante; entrevistas; análise de documentos) desenvolvida de abril a dezembro de 2011, compartilhei, com os participantes, diversos momentos de convívio, como oficinas de atividades físicas, atividades sociais, reuniões, passeios, e outras situações de convivência. Após esse longo período de produção de dados, examinei o material obtido, desenvolvi categorias de análise e cheguei a interpretações que foram apresentadas em diferentes tópicos/aspectos/temáticas que me ajudaram a responder às perguntas do estudo, e que se relacionaram: ao sentimento de pertencimento; às perspectivas de corpo e saúde presentes naquele contexto; e às lógicas de sociabilidade que sustentavam as dinâmicas sociais daquele universo. Sobre pertencimento, compreendi que vivenciar a velhice no CELARI significa compartilhar um ambiente predominantemente feminino onde o sentimento de pertencer permeia as relações cotidianas. Observei que a concepção de saúde dos idosos ultrapassa a condição biológica e se relaciona com felicidade, divertimento e sentir-se bem naquele contexto. Pude perceber que a preocupação com o corpo e com a boa forma são aspectos que atravessam o processo de envelhecimento. Porém, se essa preocupação se vincula predominantemente a uma dimensão utilitária do corpo (saúde biológica; autonomia), ela também se relaciona com a busca por um corpo bonito, o qual também faz parte dos objetivos dos participantes. O CELARI é, então, um espaço que viabiliza diversas formas de sociabilidade e de interação entre os participantes, onde as trocas afetivas costuram as relações cotidianas e, por vezes, sustentam a participação no projeto.

palavras - chave

Lazer. Sociabilidade. Velhice.

ZANINI, Adriana Mokwa. Evidências de validade do teste de retenção visual de benton (bvrt) em uma amostra de idosos. 2012. 49, fl. Dissertação (mestrado) Universidade Federal do Rio Grande do Sul. Instituto de Psicologia. Programa de Pós-Graduação em Psicologia, Porto Alegre, BR-RS, 2012. Ori.: TRENTINI, Clarissa Marceli. (texto não disponível on line) 
O objetivo geral dessa pesquisa foi contribuir com evidências de validade do Benton Visual Retention Test (BVRT). O Estudo 1 investigou evidências de validade do tipo convergente entre o BVRT e outros dois testes de memória visual: Figuras Complexas de Rey e Teste Pictórico de Memória (TEPIC-M). Participaram 29 idosos, de ambos os sexos, com 60 a 79 anos, e escolaridade baixa e média. O Estudo 2 verificou evidências de validade de critério do BVRT, comparando idosos saudáveis a idosos com diagnóstico possível de Doença de Alzheimer (DA). O grupo controle foi composto por 50 idosos saudáveis, enquanto o clínico contou com 16 idosos. Estatísticas descritivas e inferenciais (correlações - Estudo 1 e comparação de médias - Estudo 2), foram utilizadas para a análise dos dados. Ambos os estudos apontaram para evidências de validade do BVRT, o que permite indicar o instrumento para auxílio em diagnóstico e tratamento na área da neuropsicologia.

palavras - chave

Psicologia. Validade do teste. Idoso. Avaliação neuropsicológica. 
